type of fcc packing, in which there are two atoms in each face-centred unit cell, possessed the long-sought photonic bandgaps. Those two atoms per cell are the famous diamond structure of engagement rings, hard industrial stone and silicon electronic chips - now reinvented for electromagnetic waves and Maxwell's equations. Diamond topologies quickly led to the first working photonic-bandgap crystal $^{11}$, albeit at inappropriately named 'microwave dimensions'. These structures are not microscopic at all. They are big and easy to make in a conventional machine shop, but their complex geometry is hard to fabricate at optical wavelengths ${ }^{12,13}$.

Nature, of course, prefers to make simple fcc crystals. For example, $\mathrm{SiO}_{2}$ spheres naturally self-assemble as fcc in opal, another jewel. As we saw, simple, easy-to-make fcc crystal structures had been proposed as photonic bandgaps ${ }^{4,5}$, initially corroborat$\mathrm{ed}^{6}$, and finally rejected ${ }^{3,8,10}$. But the tables were to turn again. The periodic structure of a crystal forces the frequencies to fit into a series of allowed bands. Everyone had been looking for a forbidden bandgap in fcc between the second and third bands. Gazing higher, between the eighth and ninth bands, Joe Haus and colleagues ${ }^{14}$ discovered a true forbidden gap in fcc; it was not a large oneonly a few per cent of its central frequency but it was a forbidden gap nonetheless.

The fcc geometry preferred by Maxwell's equations consists of spherical voids that are easily made by etching away spheres in a matrix of our choosing. Busch and John chose a silicon matrix, very appropriate to our times. They then added a new ingredient by calculating what would happen if the spherical voids of the photonic crystal were partially filled with liquid crystals (Fig. 1). We recognize liquid crystals as the electrooptic display molecules in portable computers. An electric field can rotate these molecules, thereby modulating the refractive index of the twisted nematic liquid crystal from $n=1.4$ to $n=1.6$, a fairly respectable change. Busch and John show that by applying an electric field to their hypothetical material a $2 \%$ photonic bandgap may be completely opened or closed. They give this new concept the rather glamorous name of a 'tunable electromagnetic vacuum'. Electromagnetic fluctuations are indeed being tuned in and out of existence, but they exist within tangible materials like silicon and liquid crystal molecules, rather than a vacuum.

In fact, as much as $40 \%$ of Busch and John's proposed photonic structure is a vacuum. To work correctly, the spherical voids must have their internal surfaces coated by the liquid-crystal molecules, so becoming half filled and leaving voids that may be difficult to achieve in practice. Nonetheless, Busch and John have linked forever the fields of liquid and photonic crystals. For example, such materials could lead to light-emitting diode displays with pixels whose colours can be altered.

It is possible that the liquid/photonic crystal idea might bear fruit in structures even simpler than the three-dimensional fcc crystals. Two-dimensional photonic crystal semiconductor films have recently made the tiniest lasers, and probably the tiniest electromagnetic cavities, ever ${ }^{15}$. In conjunction with liquid crystals, they can be subject to extraordinarily delicate control, over lasing and other functions. Among possible applications, we may find photonic crystal lasers ${ }^{1}$ in tiny optical integrated circuits, or we may see photonic crystals performing as simple white pigments or as radio wave structures in wireless information appliances, such as palmtop computers and sophisticated mobile phones ${ }^{2}$.

Eli Yablonovitch is in the Department of Electrical
Engineering, University of California, Los Angeles, California 90095-1594, USA.

e-mail: eliy@ucla.edu

. Scherer, A. et al. (eds) IEEE J. Lightwave Technol. (special issue on electromagnetic crystal structures) (in the press).

Scherer, A. et al. (eds) IEEE Trans. Microwave Theory Techniques (special issue on electromagnetic crystal structures) (in the press).

3. Busch, K. \& John, S. Phys. Rev. Lett. 83, 967-970 (1999).

4. Yablonovitch, E. Phys. Rev. Lett. 58, 2059-2062 (1987).

5. John, S. Phys. Rev. Lett. 58, 2486-2489 (1987).

6. Yablonovitch, E. \& Gmitter, T. J. Phys. Rev. Lett. 63, 1950-1953 (1989).

7. Leung, K. M. \& Liu, Y. F. Phys. Rev. Lett. 65, 2646-2649 (1990).

8. Zhang, Z. \& Satpathy, S. Phys. Rev. Lett. 65, 2650-2653 (1990).

9. Maddox, J. Nature 348, 481 (1990).

10. Ho, K. M., Chan, C. T. \& Soukoulis, C. M. Phys. Rev. Lett. 65, 3152-3155 (1990)

11. Yablonovitch, E., Gmitter, T. J. \& Leung, K. M. Phys. Rev. Lett. 67, 2295-2298 (1991).

12.Lin, S.-Y., Fleming, J. G., Sigalas, M. M., Biswas, R. \& Ho, K. M. Phys. Rev. B 59, R15579- R15582 (1999)

13. Noda, S., Yamamoto, N., Kobayashi, H., Okano, M. \& Tomoda K. Appl. Phys. Lett. 75, 905-907 (1999).

14. Sozuer, H. S., Haus, J. W. \& Inguva, R. Phys. Rev. B 45, 13962-13972 (1992).

15. Painter, O. et al. Science 284, 1819-1821 (1999).

\title{
Photosynthesis \\ Stealing the limelight on the forest floor
}

By only opening their stomata

- the pores in their leaves

through which carbon dioxide

enters and water leaves - at

night, and thus achieving a

temporary carbon dioxide fix

using the enzyme phosphoenol

pyruvate carboxylase, so-called

CAM plants are able to minimize water loss. Not surprisingly, they are typically associated with arid environments. But a question mark hangs over certain bromeliads (members of the pineapple family) that grow in the deeply shaded understorey of Central American rainforests. Such moist, low-light

environments seem inappropriate for CAM plants, but the work of John Skillman et al. has shown that they occupy a niche in which high light

tolerance and drought resistance have unexpected advantages (Ecology 80,

1584-1593; 1999).

Most of the CAM plants in rainforests are epiphytes, growing perched on the branches of trees. Here, without access to groundwater, CAM metabolism helps them to survive occasional hot, dry spells. But there are also CAM plants growing in the shady understorey. Aechmea magdalenae, pictured here, is a ground-dwelling bromeliad that grows in a range of habitats in

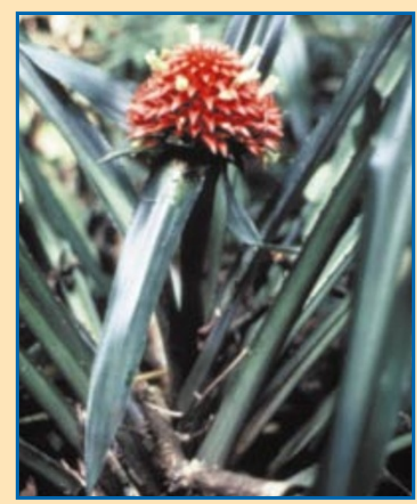

found that experimental artificial sunflecks produced a much stronger response in Aechmea than that found in $\mathrm{C}_{3}$ plants from the same habitat, accompanied by a shorter lag phase for the plant to adjust to the new light conditions.

They also found that Aechmea allocates less energy to root formation, and grows more slowly, than associated $\mathrm{C}_{3}$ plants. But it can gain an advantage over other plant species during the dry season. When soil water

Central America, from welldrained, open locations to moist, shaded sites on the forest floor. Skillman and Winter previously found that Aechmea is capable of high photosynthetic rates under bright light (Plant Physiol. 113, 441-450; 1997). But how can it compete in the shade particularly as CAM plants are less efficient photosynthesizers than ' $\mathrm{C}_{3}$ ' plants (the most widespread form of plant metabolism) where light levels are low?

One possible explanation is that Aechma is particularly adept at exploiting the occasional patches of high-intensity sunlight ('sunflecks') that penetrate the dense canopy of the forest. Using chlorophyll fluorescence, which records the energy-trapping stage of photosynthesis, Skillman et al. becomes scarce, the wate conservation properties of the bromeliad, resulting from its tough leaves and its capacity to keep its stomata closed in the daytime, ensure survival and continued growth. Aechmea grows best during the dry season, whereas the $\mathrm{C}_{3}$ plants grow best in the wet season. Although the forest floor is generally shady and moist, there is clearly a niche here for a plant that can take advantage of brief periods of high light and low water availability. Even among shade plants, there are some destined to grab the limelight. Peter D. Moore

Peter D. Moore is in the Division of Life Sciences, King's College, Franklin-Wilkins Building, 150 Stamford Street, London SE1 8WA, UK. e-mail:peter.moore@kcl.ac.uk 